\title{
Microtubules During the Cell Cycle of Higher Plant Cells
}

\author{
Elena Smirnova \\ Biology Faculty, Lomonosov Moscow State University, \\ Russia
}

\section{Introduction}

The microtubules control many cellular functions and play a key role in cell morphogenesis and development. They are dynamic heteropolymers composed of $a-$ and $\beta$-tubulin and microtubule associated proteins (MAPs) that bind to microtubules and regulate their polymerization, organization and dynamic behavior. The structure of microtubules and tubulins are almost identical from yeast to human, yet the mechanisms that control the assembly, organization and behavior of microtubules, vary among different organisms. Thus, in animal cells the microtubule system is usually organized by the centrosome, the dominant microtubule organizing center (MTOC) that nucleates microtubules and controls spatial organization of the microtubules in the cell. Microtubule minus ends are anchored at MTOC, while the dynamic plus ends are oriented towards the cell periphery, forming asterlike array. Interphase cells typically assemble single "aster" with long microtubules often reaching the cell periphery. Upon the entry to mitosis, the spindle, composed of two asters with short microtubules, replaces interphase aster-like array. Unlike animal cells, plants do not have centrosome-like MTOC and assemble several distinct microtubule arrays, replacing each other during the cell cycle. The cortical microtubule system (CMT) is present during interphase (G1, S, and G2 phases) and plays a crucial role in the construction of the cell wall by guiding the deposition of new cell wall polymers. In many types of cells microtubules emanate from the nuclear surface towards the cell cortex, assembling another interphase array termed radial microtubule system (RMT). During cell division, microtubules rearrange into specialized arrays - the preprophase band (PPB), the spindle and the phragmoplast. The PPB and phragmoplast are unique to plants. The PPB (a circular array of microtubules) forms in G2 phase and disassembles prior to the nuclear envelope breakdown at the onset of prometaphase. The PPB defines the future division plane and the site of the cell plate formation during cytokinesis. The plant mitotic spindle provides equal distribution of chromosomes in mitosis, yet spindle assembly and organization differ from those in other eukaryotes, mainly due to the absence of the defined centrosome/MTOC in the spindle poles. The phragmoplast is a special microtubule array that substitutes the contractile ring of animal cells during cytokinesis. Phragmoplast directs the synthesis of a new cell wall that physically separates two daughter cells. Different microtubule arrays have distinctive features, use different tubulin isoforms, tubulin modifications and microtubule associated proteins in assembly of each array. In view on extensive studies of the molecular mechanisms underlying the cytoskeletal functions, this chapter will be focused both on specificity, and basic structural and functional aspects of organization of plant microtubule system. 


\section{Specific features of plant microtubules}

\subsection{Tubulin}

Although tubulin $\alpha$ and $\beta$ subunits have been highly conserved over the evolution of eukaryots, plant tubulin is immunologically and pharmacologically different from tubulin of animals and fungi. Thus, animal and plant tubulin have $79-81 \%$ sequence identity; the $\alpha$ subunit of plant tubulin have distinct peptide mapping pattern, its electrophoretic mobility is higher than of $\beta$ subunit, what is in contrast with animal tubulin subunits (Fosket \& Morejohn, 1992). Plant $\gamma$-tubulin shares $60 \%$ sequence identity with animal $\gamma$-tubulin, and, oppositely to animal cells, its electrophoretic mobility is higher than of $\alpha$ and $\beta$ subunits (Liu et al., 1994). Nevertheless, microtubules comprising each plant array have an identical structure, and their functional diversity within the same cell is supported by multiple tubulin modifications. The heterogeneities of tubulin molecules are created on different levels by two mechanisms. On the genome level it is the activity of the multi-genes families, encoding plant $\alpha$ and $\beta$ tubulins. Tubulin genes are not expressed uniformly during plant development and the expression of tubulin isotypes is tissue-specific. On the cytoplasmic level it is the posttranslational tubulin modifications. Tubulin is subjected to the detyrosination/tyrosination cycle, the removal of penultimate glutamate, polyglutamylation, polyglycylation, acetylation, phosphorylation and palmitoylation. Most modifications occur on microtubules rather than on unpolymerized tubulin, and notably contribute to the diversity of tubulins. Post-translational tubulin modifications create subpopulations of microtubules, involved in specific functions within particular cellular compartments, and play an essential role in the reorganization of the microtubule cytoskeleton during the life cycle of plant cells (Cai, 2010). Thus the development of specific plant cells and tissues is characterized by the expression of distinct tubulin genes and, consequently, by the use of distinct tubulin isotypes, which are post-translationally modified to control the microtubule properties and functions.

\subsection{Resistance to microtubule depolymerizing compounds}

Plant tubulin is pharmacologically different from tubulin of animals, fungi and slime molds. This is manifested by a low affinity to colchicine, an alkaloid produced by several plants of the Liliacea family. The drug is known as an anti-microtubule and anti-mitotic agent for both plant and vertebrate cells (Eigsti \& Dustin, 1955). There are principal differences in the response of these organisms to colchicine. The minimal concentration required for mitotic block of animal and plant cells differs considerably. Thus, animal cells are about 100,000 more sensitive to the colchicine than plant cells. Colchicine is usually lethal to dividing animal cells even at the minimal concentrations necessary to block mitosis, yet plant cells can survive in a state of colchicine-induced mitotic arrest (C-mitosis) for several days and return to division after colchicine is removed (Eigsti \& Dustin, 1955). It appears that plant microtubules as compared to vertebrate ones, are more resistant to colchicine treatment due to the low affinity of plant tubulin dimers to the drug, although they can be completely disrupted by extremely high concentrations of colchicine. Dinitroaniline herbicides (oryzalin, trifluralin), amiprophosmethyl (APM) and phenylcarbamates efficiently bind to plant tubulin and prevent microtubule polymerization. Plant tubulin has a high affinity to these herbicides, which, in turn, have no effect on animal tubulin; therefore these agents are broadly used in experimental studies of plant microtubule dynamic and function (Morejohn \& Fosket, 1991). 


\subsection{Microtubule nucleation}

Higher plant cells do not have a single dominant MTOC comparable to the centrosome of animal cells or spindle pole body of yeast. Instead, plant microtubules are initiated from dispersed nucleating sites - the $\gamma$-TuRCs ( $\gamma$-tubulin ring complexes), which serve as the microtubule nucleation units. $\gamma$-TuRCs are composed of $\gamma$-tubulin small complexes $(\gamma$-TuSCs) and several other proteins. The conserved $\gamma$-TuSCs contain two molecules of $\gamma$-tubulin and one molecule each of Spc97 and Spc98 homologs, and are the minimal nucleation unit (Erhardt et al., 2002). Additional proteins in the larger ring complexes considerably enhance the nucleation activity, and some specific subunits are presumed to recruit the nucleation complexes to the particular cellular sites. $\gamma$-tubulin dependent nucleation of microtubules in plant cells occurs preferentially along the sidewalls of assembled microtubules, with new ones arising at discreet angles relative to assembled microtubules, and forming a Y-type or branched structure. A cytoplasmic $\gamma$-tubulin complex shuttles between the cytoplasm and the sides of microtubules and has nucleation activity only when bound to the microtubules.Thus microtubule nucleation in plant cells requires both existing microtubules and the presence of $\gamma$-tubulin (Murata et al., 2005).

\subsection{Microtubule associated proteins (MAPs)}

MAPs in plants regulate the assembly, repositioning and dismantling of all microtubule arrays throughout the cell cycle, and facilitate microtubule growth, dynamics, organization, and function (Sedbrook, 2004). Some MAPs are specific only to plants, while others are found in other organisms.

\subsubsection{MOR1 (microtubule organization 1)}

The Arabidopsis MICROTUBULE ORGANIZATION 1 MOR1/GEM1 is a homolog of XMAP215/TMP200 and belongs to a highly conserved MAP215/Dis1 group of MAPs found in all eukaryotes. MOR1 is essential for the organization of CMT array, proper organization and function of the PPB, the mitotic spindle, the phragmoplast formation and the progression of cell division. MOR1 and several other MAPs also contribute to microtubuleassembly dynamics (Kaloriti et al., 2007).

\subsubsection{Plus end interacting proteins (+TIPs)}

+TIPs are a diverse group of proteins characterized by their preferential localization to microtubule plus ends, where they regulate microtubule dynamic instability and mediate microtubule interactions with intracellular components such as the cell cortex, organelles, kinetochores, and other cytoskeletal elements (Bisgrove et al., 2004). Two important +TIPs in plants are END BINDING1 (EB1) and SPIRAL1 (SPR1). EB1 proteins are highly conserved in animals and fungi, yet SPR1 appears to be plant specific. Among known functions of EB1 proteins are the promotion of microtubule polymerization, stabilization, regulation of spindle positioning and chromosome segregation (Komaki et al., 2010). SPR1 and EB1 may act together to regulate directional cell expansion in response to the environmental stimulation. Another +TIP protein, CLASP, found in Arabidopsis, is involved in modulation of microtubule-cortex interactions and contributes to the PPB formation (Wasteneys \& Ambrose, 2008). 


\subsubsection{Katanin}

Katanin is a heterodimeric microtubule severing protein composed of an ATPase $60 \mathrm{kDa}$ catalytic subunit with microtubule fragmenting activity and an $80 \mathrm{kDa}$ regulatory subunit. The Arabidopsis katanin homolog exhibits a punctate localization pattern at the cell cortex and the perinuclear region. Overexpression of p60 katanin in Arabidopsis causes abnormal cortical microtubule bundling and fragmentation along their lengths. Plant katanin may function by severing microtubules at their minus ends, thus releasing the minus ends from the nucleating centers and allowing microtubules to be organized into bundles (Kaloriti et al., 2007).

\subsubsection{MAP65 family}

MAP65 family is a group of $60-65 \mathrm{kDa}$ proteins that co-purify with microtubules. The Arabidopsis genome encodes nine MAP65 proteins of varying functions. Arabidopsis, tobacco, zinnia and carrot homologs bundle microtubules in vitro and, in some instances, in vivo. Some MAP65 members (AtMAP65-1, AtMAP65-3, NtMAP65-1a) belong to midzone MAPs because they localize at the antiparallel region of the developing phagmoplast and probably play essential role in cytokinesis by crossbridging microtubules that need to retain spatial organization in reorganizing microtubule arrays (Kaloriti et al., 2007). MAP65-4 regulates dynamic instability of microtubules by decreasing catastrophe and increasing rescue events. It is colocalized with mitotic microtubules, specifically with microtubules of the developing mitotic spindle during prophase and with the kinetochore fibers from prometaphase to the end of anaphase. Thus, MAP65- 4 could mediate lateral interactions between spindle microtubules and participate in the formation and dynamics of microtubules within kinetochore fibers (Fashe et al., 2010).

\subsubsection{Wave-dampened 2}

WVD2 gene encodes a $23 \mathrm{kDa}$ MAP that appears to regulate cell expansion through its association with and/or organization of cortical microtubules. WVD2 C-terminal domain is distantly related to the vertebrate microtubule-associated protein TPX2, involved in RanGTP-mediated spindle assembly around chromosomes.

\subsubsection{AIR 9 (auxin induced root cultures 9)}

AIR9 is a $187 \mathrm{kDa}$ MAP, conserved in plants and found on microtubules of the cortical array and the PPB, but down regulated during mitosis. AIR9 reappears at the former PPB site when growing phragmoplast contacts the cortex, at the site of cell plate insertion and on the new cross walls, suggesting that AIR9 recognizes a component of the former PPB. Thus AIR9 may be involved in the maturation of those cell plates, which already contacted the established by the PPB cortical division site (Buschmann et al., 2006).

\subsubsection{AtMAP70}

MAP70 is a plant specific multi-gene family of proteins, sharing $70-80 \%$ identity and approximately $70 \mathrm{kDa}$ molecular mass. AtMAP70-1 colocalizes with microtubules of all arrays, but is missing in microtubules of the midzone. AtMAP70-5 is smaller (58 kDa), 
shares $47 \%$ sequence identity, and its possible functions are the regulation of microtubule dynamics and a role in anisotropic cell expansion and organ growth (Kaloriti et al., 2007).

\subsection{Plant motors}

Microtubule motor proteins play an essential role in the organization and function of microtubule arrays during cell division and cell growth in plants. They are responsible for the motility of macromolecular complexes and organelles, and the segregation of chromosomes during mitosis and meiosis.

\subsubsection{Kinesins}

61 genes encoding kinesins were identified in the genome of Arabidopsis thaliana, and most kinesins are evolutionarily divergent from their counterparts in animals and fungi (Lee \& Liu, 2004). Kinesins are grouped into sub- families by phylogenetic analyses of their motor domains.

Plus end directed kinesins:

1. The N-terminal motor kinesin AtFRA1 (kinesin-4) belongs to the kinesin-4 or KIF4/chromokinesin subfamily. Animal chromokinesins typically play roles in chromosome condensation, chromatid motility and microtubule assembly, while AtFRA1 contributes to cellulose microfibril deposition in the cell wall. Thus the sequence similarity in the motor domain does not necessarily confer any functional relationship among kinesins from different organisms.

2. The internal motor kinesin - kinesin-13. Two Arabidopsis kinesins, AtKinesin-13A and AtKinesin-13B, belong to the kinesin-13 subfamily, previously known as MCAK or KIN I. AtKinesin-13A is specifically associated with plant Golgi stacks. Animal internal motor kinesins in this subfamily are not motors. Instead, they are microtubule depolymerases activated by microtubule end binding. Therefore, this again demonstrates that sequence homology in the motor domain alone does not guarantee functional similarity for kinesins from different organisms.

3. Kinesins in the BIMC (Kinesin-5) subfamily. Four Arabidopsis genes encode kinesins in the BIMC/Kinesin-5 subfamily. Besides the sequence conservation in the motor domain of these four kinesins and their animal and fungal counterparts, all kinesin-5 members contain a conserved phosphorylation site for the key cell-cycle kinase p34cdc2.

Minus end directed kinesins:

A number of C-terminal kinesins are minus end-directed motors. Among minus enddirected kinesins, however, some have the motor domain located at the N-terminus, while some others have it in the middle.

1. KATA/ATK1 and close relatives. These kinesins are closely related to the NCD (nonclaret disjunction) kinesin-14 subfamily and are implicated in the organization of microtubules of the spindle and the phragmoplast.

2. The calmodulin-binding KCBP/ZWI kinesin. KCBP/ZWI is a single gene that encodes a unique calmodulin-binding kinesin, possibly involved in stabilization of microtubule ends. 
3. The actin-binding $\mathrm{KCH}$ kinesins. Minus end-directed kinesins with a unique calponinhomology $(\mathrm{CH})$ domain at the $\mathrm{N}$ terminus. They have only been reported in organisms of the kingdom Plantae. The presence of a $\mathrm{CH}$ domain in a kinesin is intriguing as it is typically found in actin-binding proteins like calponin and fimbrin. $\mathrm{KCH}$ s may serve as linkers between microtubules and actin microfilaments.

4. Other minus end-directed kinesins. There are nine other Arabidopsis kinesins in the category of minus end-directed motors. Four have the motor domain located at the $\mathrm{N}$ terminus, the feature that has never been reported for kinesins from animals and fungi.

Special group of kinesins involved in cytokinesis:

N-terminal kinesins are involved in cytokinesis and localize to the division site, yet in distinct manner.

1. AtNACK1/HIK kinesins and a MAP kinase cascade. AtNACK1/HIK is an activator of a mitogen-activated protein (MAP) kinase cascade and is essential for the completion of the cell plate formation. NACK1 is also required for cell plate expansion after initiation of the cytokinesis.

2. AtPAKRP1/AtKinesin-12A and similar kinesins. AtPAKRP1/AtKinesin-12A and two similar kinesins are specifically associated with the plus end of phragmoplast microtubules and are predicted as plus end-directed motors. They are implicated in the maintaining of orderly organization of microtubules within the phragmoplast.

3. Kinesins transporting Golgi-derived vesicles. Phragmoplast-associated kinesin, AtPAKRP2, is specifically associated with Golgi-derived vesicles in the phragmoplast. At least two other Arabidopsis kinesins are also exclusively associated with vesicles in the phragmoplast. Therefore, they are predicted to be examples of plus end-directed kinesin motors that deliver Golgi-derived vesicles during cytokinesis.

\subsubsection{Dynein}

Higher plants (angiosperms) lack cilia and flagella in their life cycle, and their non-motile sperm cells are conveyed to the egg by a growing pollen tube. These plants also lack the axonemal dynein, cytoplasmic dynein 2 and no full-length genes for cytoplasmic dynein 1 subunits, except for the light chain LC8, have been found in the sequenced genome of Arabidopsis thaliana, Oryza sativa and Populus trichocarpa. However, immunological and biochemical studies showed the presence of dynein heavy chain (DHC) related polypeptide in pollen tubes of Nicotiana tabacum (Moscatelli et al., 1998) and root cells of wheat Triticum aestivum L. (Shanina et al., 2009). Dynein is an ancient and evolutionary conservative multiprotein complex, found in diverse groups of organisms, from yeast to human, and DHC is the largest dynein subunit, containing ATP-binding/motor and microtubulebinding domain. To date, nothing is known about dynein in plants that have flagella apparatus at certain stages of their life cycle. For instance, the microtubules of the flagellar axonemes of water fern Marsilea vestita have the typical $9+2$ arrangement with only inner dynein arms present (Hyams, 1985). Flagellated sperm cells also develop in ancient gymnosperms, Ginkgo and cycads, which are the only seed producing plants (spermatophytes) that have motile or free swimming sperm (Vaughn and Renzaglia, 2006) with abundant flagellar apparatus (100-50,000 flagella per cells) (Renzaglia and Garbary, 
2001). Thus, higher plants have high molecular weight antigen that shares biochemical and immunological properties with DHC, yet its identity remains unclear.

\subsection{Microtubule dynamics}

The distinctive feature of plant microtubules is the unusual dynamic behavior, exemplified at least in some stages of microtubule life cycle. Cortical microtubules in plant cells exhibit the treadmilling (addition of tubulin subunits at plus ends and removal at minus ends), what is remarkably different from the behavior of microtubules in animal cells, when microtubules are attached to the centrosome with their minus ends, and plus ends exhibit the dynamic instability (slow growth - rescue, and rapid disassembly - catastrophe). Moreover, plant microtubules display a unique type of dynamics, a combination of dynamic instability and treadmilling behavior, termed "hybrid treadmilling", which involves dynamic instability behavior at the plus end, coupled with a slow depolymerization at the minus end. Polymerization-biased dynamic instability at one end and slow depolymerization at the other end results in sustained microtubule migration across the cell cortex (Shaw et al., 2003).

Another unique feature of higher plant microtubules is that unlike microtubules of most animal cells, they do not originate from a single or multiple MTOCs (Mineyuki, 2007; Murata et al., 2007). Plant microtubules change the arrangement with the progression of the cell cycle, assembling distinct types of microtubule arrays, which function at the appropriate stage of the cell cycle. The CMT system or/and RMT system is present during interphase, $\mathrm{PPB}$, mitotic spindle and phragmoplast successively replace each other during mitosis (Fig. 1, 2).
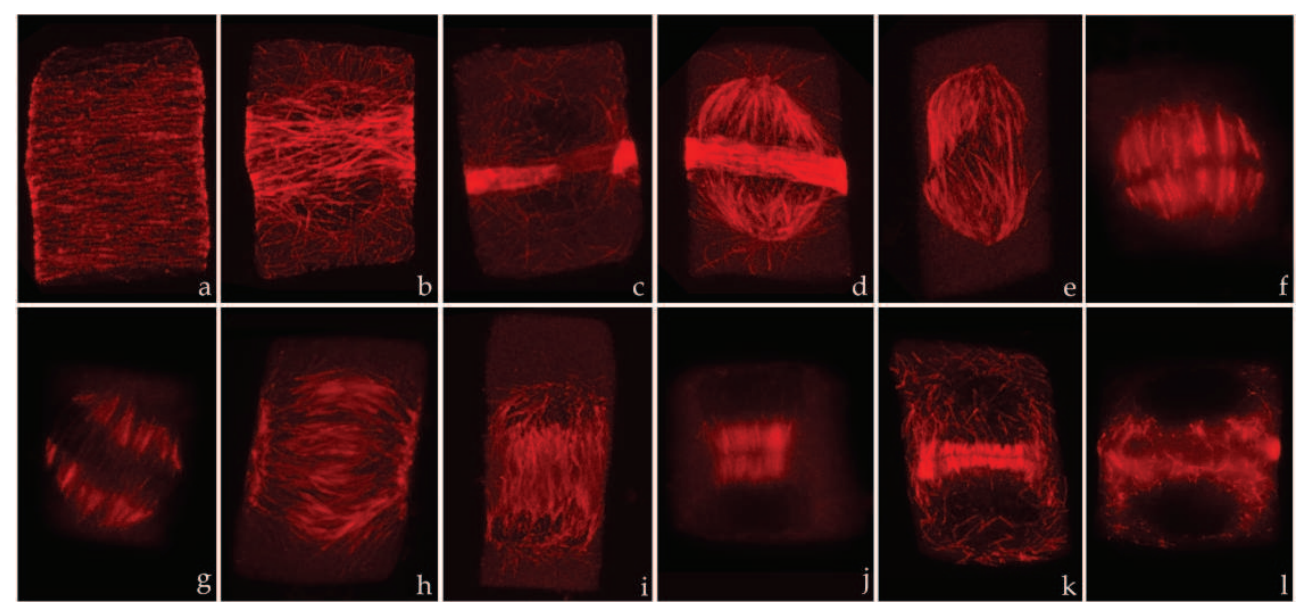

Fig. 1. Microtubules during the cell cycle of root meristem cells of Triticum aestivum L. (a) Cortical microtubule bundles in interphase cell. (b) The development of broad PPB during G2 phase. (c) Matured PPB in prophase cell. (d) PPB and prophase spindle. (e) Prometaphase spindle. (f) Metaphase spindle. (g) Anaphase spindle. (h) Initial stage of phragmoplast formation in anaphase. (i-k) Successive stages of phragmoplast formation and development during anaphase-telophase. (k-1) Radial microtubule network during transition to G1 phase. 

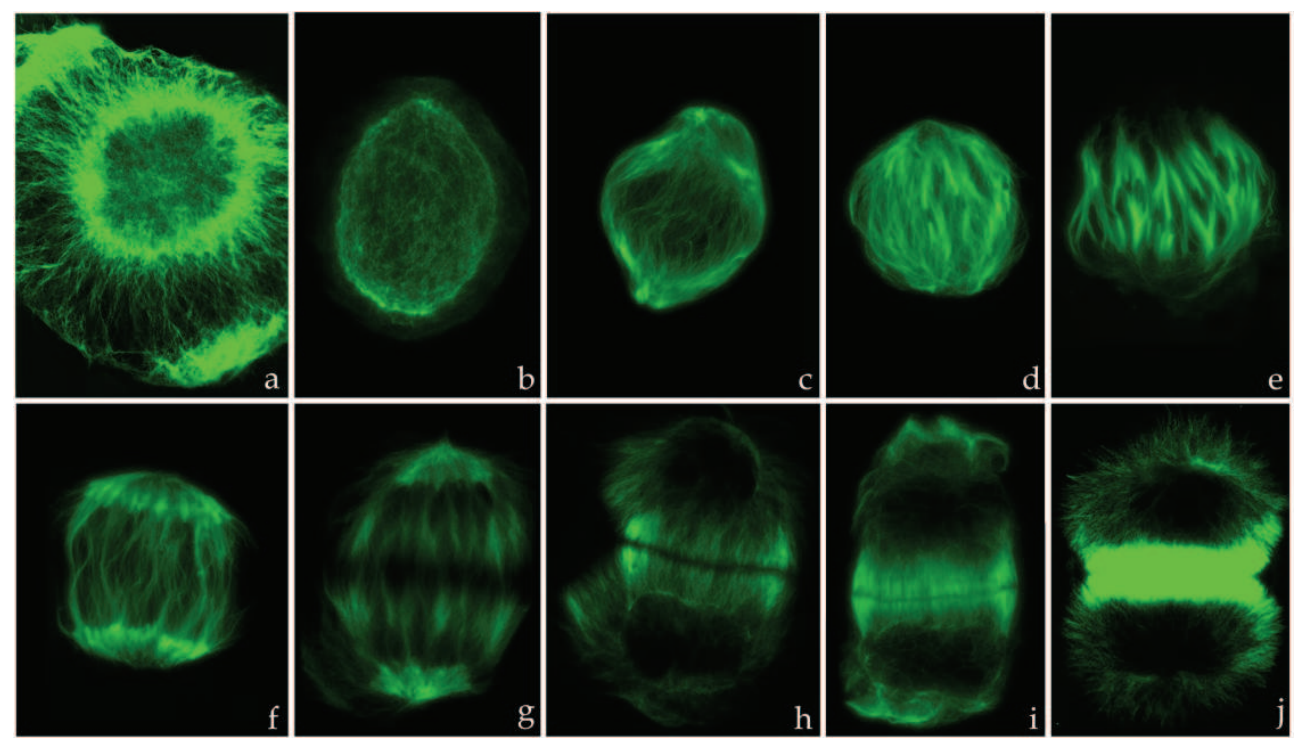

Fig. 2. Microtubules during the cell cycle of isolated endosperm cells of Scadoxus katherinae Bak. (a) Radial microtubule system in interphase cell. (b) Early stage of prophase spindle organization. (c) Prophase spindle. (d) Prometaphase spindle. (e) Metaphase spindle. (f, g) Spindle in mid and late anaphase. ( $h$, i) Phragmoplast formation and development during telophase. (j) Radial microtubule arrays during transition to G1 phase.

\section{Microtubule systems of interphase cells}

\subsection{Radial microtubule system (RMT)}

RMT system is composed of microtubules radiating from the nuclear surface toward the cell periphery. During somatic division of flowering plants this microtubule array is observed for relatively short time, when the cell is completing the cytokinesis and before the establishment of the interphase CMT (Fig. 1k-1). RMT system is typical for some special cases of flowering plant cell division (Fig. 2a, j). In isolated endosperm cells of Scadoxus, RMT system is composed of elementary structural units - microtubule converging centers (MTCCs). The pointed tips of MTCCs are associated with the nuclear surface, and the divergent ends are oriented outwards and extend toward the plasma membrane. Cumulative evidence indicates that minus end of radial microtubules are associated with the nuclear surface, while plus ends are oriented toward the cell periphery. MTCCs could be the transitory structures, instrumental for orderly reorganization of RMT and other microtubule arrays in plant cells (Smirnova \& Bajer, 1994, 1998). RMTs arrays emanating from the nuclear surface are also present in vacuolated cells, where they are termed endoplasmic microtubules (EMTs) (Dhonukshe et al., 2005). Thus nuclear surface may function as MTOC for RMTs, yet the microtubule nucleating factors have not been clearly identified. Chromatin affects microtubule nucleation through the nuclear membrane, as it was shown for reconstituted nuclei in Xenopus extracts (Heald et al., 1996, 1997). Targeting protein for Xklp2 (TPX2) is one of the proteins implicated in the nucleation of microtubules around chromosomes in a RanGTP-dependent manner. TPX2 orthologue was found in Arabidopsis, (Evrard et al., 2009) and experimental studies showed that it nucleates 
perinuclear microtubules/RMTs, leading to the formation of a prophase spindle, and then may act as a spindle microtubule nucleator and stabilizer (Vos et al., 2008).

\subsection{Cortical microtubule system (CMT)}

Plant CMT network consists of a population of relatively short, overlapping microtubules that bundle into a higher order structures. In rapidly elongating cells, the CMT bundles typically circumference the cell and are arranged transverse to the elongation axis of the cell (Fig. 1a). Once the phase of cell elongation slows down, the transverse CMTs reorient into a predominantly oblique or longitudinal direction with respect to the cell's elongation axis (Wasteneys, 2004; Lucas \& Shaw, 2008; Wasteneys \& Ambrose, 2008).

Several mechanisms have been proposed in order to explain the assembly and dynamic organization of the cortical arrays:

1. Microtubules are initiated de novo or originate from the existing microtubules in the cortical cytoplasm. First, seed microtubules appear at the cortex of early interphase cells. Then $\gamma$-tubulin nucleates new microtubules along the lengths of existing microtubules, resulting in dispersed ' $Y$ '-branched organizational centers (Murata et al., 2005). As a result, new cortical microtubules form on the pre-existing microtubules as branches with a defined angle (Hashimoto \& Kato, 2006). Nascent microtubules detach form the nucleation sites and migrate to the cortex by "hybrid" treadmilling (Shaw et al., 2003).

2. Microtubules originate from the nuclear surface, then are severed and translocated to defined cortical positions, establishing CMT. At the M/G1 transition, microtubules are nucleated on the nuclear surface and extend to the cell cortex. $\gamma$-tubulin also first accumulates at the nuclear surface and then spreads to the cortex. Therefore, the radial/endoplasmic microtubules could be involved in distributing the $\gamma$-TuRCs from the nuclear surface to the plasma membrane. However, the majority of new microtubules are initiated de novo at the cell cortex and do not originate from the endoplasmic microtubules. New microtubule initiation sites have been detected only in associations with pre-existing microtubules and in cortical regions that have no detectable microtubules (Hashimoto \& Kato, 2006; Wasteneys \& Ambrose, 2008).

3. Lateral and axial sliding (translocation) of existing microtubules into new positions results in relocation, rearrangement and redistribution of microtubules in the cortex and/or from the perinuclear location toward the cortical cytoplasm. After microtubules are nucleated and released/severed from $\gamma$-TuRCs, both ends of the microtubules are free. The assembly dynamics of free microtubules may be marked by net polymerization at the plus-ends and net depolymerization at the minus-ends. The combined effect of these dynamic properties produces sustained treadmilling motility, when single microtubule translocates across the cell cortex. Thus, the migration of polymers to new positions is achieved by the balanced addition and removal of subunits at the microtubule ends.

\subsubsection{Dynamic behavior of microtubules in the cortex}

CMTs arise or arrive in random orientations throughout the cell's cortex, and this orientation is maintained in actively dividing meristem cells. In rapidly elongating cells, the discordant microtubule orientations do not persist for long, and microtubules of cortical arrays tend to acquire a common polarity by selective stabilization of concordant microtubules. Treadmilling leads to microtubule interactions, resulting in microtubules crossing over one another, 
selectively disassembling, pausing or bundling. The latter depends on the angle of interaction between encountering microtubules. A steep angle of interaction (more than $40^{\circ}$ ) usually leads to the disassembly of the microtubules, while the shallow angle (less than $40^{\circ}$ ) results in alignment and lateral interaction of the encountering microtubules (Ehrhardt, 2008). MAPs, including +TIPs and those forming the cross-bridges between the lattices of adjacent microtubules (MAP65 family), may be involved in the organization of microtubule bundles. The combination of microtubule encounter activities ultimately determines the spatial organization of cortical microtubules in each cell types and explains the mechanism of selforganization of randomly nucleated microtubules into parallel arrays (Pastuglia \& Buchez, 2007; Lucas \& Shaw, 2008; Wasteneys \& Ambrose, 2008).

\subsubsection{Association of cortical microtubules with plasma membrane}

After microtubules are initiated in the cortical cytoplasm, they usually detach from the original nucleation sites but remain tightly anchored to the cortex. The presence of crossbridge structures between cortical microtubules and the plasma membrane points to the presumptive plasma-membrane-associated protein linkers anchoring microtubules to the cortex. Phospholipase is one of the molecules involved in attaching the CMTs to the plasma membrane (Kaloriti et al., 2007), yet cortical microtubule attachment could be also mediated by the +TIPs.

\subsubsection{The functions of CMT array}

Most plant cells have cellulose cell walls and CMT network beneath the plasma membrane. During interphase, CMTs are typically coaligned with the cellulose microfibrils, and, therefore, it has long been thought that the organization of the interphase CMTs regulates the axis of cell elongation by guiding the oriented deposition of cellulose microfibrils. The microfibrils, in turn, provide the constraints to restrict turgor-induced cell expansion to an axis perpendicular to the net orientation of the cellulose microfibrils. The functional association of microtubules with the cellulose synthase complex located on the plasma membrane (Paredez et al. 2006) strongly supports the concept that at least in some cell types, cortical microtubules can organize cellulose synthase complexes and guide their movement through the plasma membrane as they create the cell wall. The latest data confirmed that cortical microtubules not only guide the trajectories of cellulose synthase complexes in the plasma membrane, but also regulate the insertion and internalization of cellulose synthase complexes (Crowell et al., 2009). Moreover, cellulose synthase complexes also influence cortical microtubule array stability and organization (Paredez et al., 2008), indicating that while cortical array organization directs the trajectories of cellulose synthase complexes movement, the activity of cellulose synthase complexes affects cortical array organization as well. However, organized cortical microtubules are not always essential for maintaining or establishment of transversely oriented cellulose microfibrils in expanding cells, indicating that the relationship between CMT array organization, microfibril orientation and the axis of cell elongation is more complex. In this view, the template incorporation model and microfibril length regulation model are the alternative concepts of microtubules-microfibrils interplay (Wasteneys, 2004).

\section{Preprophase band of microtubules (PPB)}

Most differentiated and expanded cells have a system of parallel bundled microtubules oriented perpendicular to the longest axis of the cell and localized in the cortical cytoplasm 
(CMTs). Prior to mitosis, this cortical system is replaced by the PPB, a unique plant array of cortical microtubules and actin filaments, encircling the nucleus and positioned just underneath the plasma membrane. PPB is formed in G2 phase and disassembled prior to prometaphase. The PPB at first occupies broad area of the cortex (about 2/3rd of the peripheral area), but then it gradually "matures", microtubules, forming the band, become more densely packed as the cell approaches the mitosis (Fig. 1b, c). PPB demarcates the cortical division site where the newly formed cell plate will be fused with the parental plasma membrane (Van Damme et al., 2007, Van Damme \& Geelen, 2008; Müller et al., 2009).

\subsection{The occurrence of PPB}

The division events that involve a PPB is thought to be an adaptation for cells that are part of a complex multicellular architecture. Indeed, PPBs occur in meristems and meristemoids during vegetative growth, and absent in microsporogenesis, megasporogenesis, in the first assymetrical mitosis of the embryo, in embryo sac development (Mineyuki, 1999). In line with the idea that the PPB correlates with divisions in tissues, it was noticed that some suspension-cultured cells divide without the need for a PPB or frequently produce abnormal PPBs, but still manage to divide.

\subsection{The origin of PPB}

Few mechanisms have been proposed to explain the origin of the PPB: de novo assembly of PPB microtubules, recruitment of tubulin from the degraded cortical microtubules to microtubules of the PPB, rearrangement of existing cortical microtubules to the site of the PPB development. Current models implicate the distinctive dynamic behavior of cortical microtubules in PPB formation (Ehrhardt, 2008; Wasteneys \& Ambrose, 2008; Müller et al., 2009). During interphase, microtubules are distributed throughout the cell cortex. The growing plus ends of dynamic microtubules that are bound to the cell cortex frequently run into other single microtubules and microtubule bundles. Steep-angle collisions promote microtubule shrinkage, whereas shallow-angle encounters facilitate coalignment into bundles. These interactions of microtubules may promote the self-organization of dynamic microtubules into a parallel arrangement. During PPB initiation at the transition from interphase to mitosis, the growth rate and the stochastic alternating frequencies between growing and shrinking phases increase in cortical microtubules, outside the PPB. Combined with the stabilizing activities in the developing $\mathrm{PPB}$, these changes in microtubule dynamics may cause disassembly of cortical microtubules outside the PPB and accumulation of microtubules to form PPB. It has been assumed that at preprophase, cortical microtubules become restricted to the future plane of division via selective depolymerization of non-PPB microtubules and/or selective stabilization of microtubules in the PPB zone. However, the general hypothesis that bundling stabilizes the dynamic properties of the constituent microtubules was not confirmed, because no evidence that bundled microtubules are stabilized against depolymerization through changes to their dynamic properties was found.

\subsection{The regulation of PPB formation}

A variety of proteins is involved in PPB formation by differentially regulating nucleation, dynamics and stability of microtubules. Microtubule binding proteins like MOR1/GEM1 modulate microtubule dynamics and promote PPB formation. MAP65 members bundle 
microtubules by forming cross bridges between overlapping microtubules and thus could potentially stabilize PPB microtubules via bundling. +TIP family member CLASP has been implicated in PPB organization and narrowing via modulation of microtubule dynamics in the PPB zone and/or by mediating microtubule-cortex interactions. Arabidopsis signaling proteins, TON1a, TON1b and TON2, are also crucial for the formation of PPB, because plants lacking any of these proteins do not form PPBs and, as a result, have misoriented cell divisions (Müller et al., 2009).

\subsection{Functional properties of PPB}

The position of the PPB in the cortical cytoplasm forecasts the division plane and the site where the cell plate inserts into the mother wall during cytokinesis. The PPB is dismantled prior to prometaphase, therefore the separation in time between the destruction of the PPB and the insertion of the cell plate led to the idea that the PPB leaves behind a landmark that will guide the expanding phragmoplast to the site where the PPB was positioned prior to its disassembly. After the PPB is disassembled, some type of "memory" of its location must remain throughout mitosis and cytokinesis. A number of negative and positive markers of the division site have been found in the cortex (Müller et al., 2009; Rasmussen et al., 2011).

Negative markers:

1. The actin-depleted zone (ADZ) of the cell cortex. Actin microfilaments are an integral part of the plant cytoskeleton. During interphase-mitosis transition, the actin network does not disappear throughout the peripheral space like the cortical microtubules. Upon breakdown of the PPB a region devoid of actin, termed the actin depleted zone (ADZ), is formed in the cortex. Because the position of the ADZ corresponds to that of the $\mathrm{PPB}$, it too marks the division zone. It is important to note that this "negative template" remains throughout metaphase and anaphase, establishing the landmark at the plasma membrane. However, the role of ADZ as a negative marker of cortical division site remains ambiguous.

2. Cortical actin "twin peaks" - two bands of high actin density flanking the cortical division site. In this view, ADZ should be interpreted as a zone of low actin abundance rather than one with complete loss of the filaments.

3. The Arabidopsis kinesin-like protein KCA1 is another negative marker of the cortical division site, specifically the plasma membrane domain. Like cortical actin, it is locally depleted at the cortical division site during mitosis and cytokinesis, creating a KCA1depleted zone (KDZ). KDZ coincides with the position of the disassembled PPB and established ADZ. Once established, the KDZ does no longer require an intact microtubule or actin cytoskeleton and persists throughout cytokinesis.

4. Endocytic vesicles form more frequently in the PPB zone than in other areas of the cell cortex, suggesting that endocytosis could be important for establishment of the division plane.

Positive markers:

TAN and RanGAP1 have been identified as positive markers of the division plane, continuously localizing there from preprophase through the completion of cytokinesis.

1. The TAN1 gene encodes a highly basic protein that binds to the microtubules of the $\mathrm{PPB}$ and localizes in the cortical division site during mitosis and cytokinesis. TAN is 
implicated in guidance and linking of the phragmoplast to cortical division site throughout cytokineis.

2. Like TAN, RanGAP1 is recruited to the division plane, co-localizing with the PPB and remaining at the cortical division site throughout mitosis and cytokinesis. Ran-GAP1 is also localized to elsewhere in dividing cells including the nuclear envelope, spindle midzone, kinetochores and cell plate. It is not yet clear whether RanGAPs are required for PPB assembly/disassembly, or for phragmoplast guidance during cytokinesis.

3. A closely related Arabidopsis kinesins, POK1 and POK2 (phragmoplast orienting kinesins) are required for the correct localization of TAN and RanGAP1. POK1 and POK2 in combination are needed for localization of TAN to the PPB and cortical division site, suggesting that TAN becomes associated with the division plane as cargo of POK1 and POK2. By contrast, RanGAP1 does not require POK1 and/or POK2 for colocalization with PPBs, but does require these kinesins for its maintenance at the cortical division site after PPB disassembly.

4. The plasma membrane domain is defined by the localization of a plant specific protein TPLATE that has homology with coat proteins. During cytokinesis TPLATE accumulates in the cell plate and appears in the cortical division site just before cell plate fusion with plasma membrane. These finding suggests that TPLATE promotes vesicle trafficking to the cortical division site, where the cell plate contacts and fuses with plasma membrane.

Another essential PPB function is the proper positioning and orientation of the mitotic spindle. It is well known that cells lacking PPB may assemble multipolar prophase spindles, which are inevitably transformed into bipolar ones during prometaphase, after the nuclear envelope breakdown (Smirnova and Bajer, 1998). Alternatively, cells with PPB establish a bipolar cap-like organization of the prophase spindle perpendicular to the plane of division before nuclear envelope breakdown. It appeared that in vacuolated cells microtubules bridging the PPB and the prophase nucleus contribute to the bipolarity, orientation, and position of the prophase spindle by transmitting tensile forces that facilitate the organization of perinuclear microtubules from their initial random distribution, into two halves, oriented perpendicular to the PPB plane. This tension serves to co-align perinuclear microtubules in a direction similar to those of the bridge microtubules, thus providing a spatial cue for the orientation of the prophase spindle axis. Consequently, the PPB is an equatorial organizer of the prophase spindle (Ambrose \& Cyr, 2008). However, non vacuolated cells have scarce or no microtubules bridging the PPB to the nucleus, therefore other mechanisms may control the spindle polarity and orientation.

Thus, the major PPB functions are the determination of the cortical division plane, promotion of the spindle morphogenesis and positional stability. PPB also controls premitotic migration and orientation of the nucleus in the cytoplasm of vacuolated cells, anchoring the premitotic nucleus in the central cytoplasm, and is a source of tubulin/microtubules pool for the assembling spindle.

\section{Plant spindle}

The mitotic spindles of higher plants do not have microtubule focusing structures at the spindle poles therefore it is usually barrel-shaped and lacks astral microtubules (Fig. 1f, 2d). The spindle consists of distinct kinetochore fibers (K-fibers), composed of kinetochore and non-kinetochore microtubules. The proximal parts of the K-fibers are attached to the 
kinetochores, while convergent distal ends are oriented away from the chromosomes and terminate at the ill-defined polar areas. For instance, the mitotic spindle of the higher plant Scadoxus (formerly Haemanthus) is composed of multiple contiguous K-fibers, arranged as microtubule fir trees (MTFTs). Parallel central microtubules of MTFT, attached to the kinetochore, form a core of K-fiber (the trunk of the "fir tree") and non-kinetochore skew peripheral microtubules, represent the branches (Bajer and Molè-Bajer, 1982, 1986). All Kfibers/MTFTs within each half-spindle are aligned parallel to the spindle axis, and the structural integrity of the spindle is maintained by skew non-kinetochore microtubules, whereas the functional properties (support of chromosome movement) are provided by kinetochore microtubules. Each half-spindle may be composed entirely from K-fibers, conjoined together by non-kinetochore microtubules. The mitotic spindle is responsible for equal segregation of sister chromatids during cell division. This function is accomplished by the establishment of a plane of symmetry, which is materialized by the metaphase plate, orthogonal to the spindle axis and on which the chromosomes are positioned before separation.

\subsection{The flexibility of plant spindle}

Although conventional metaphase spindle is barrel-shaped and lacks astral microtubules, its shape may be altered during specialized types of division, for instance, during generative cell/nucleus division in pollen tube of different plants. In Tradescantia virginiana and Convallaria majalis, the spindle is comprised of an axial system of microtubule bundles, with kinetochores distributed along the length and depth of the tube. The same type of division in Nicotiana tabacum is characterized by oblique metaphase plate and the presence of distinct spindle with often pointed poles (Liu \& Palevitz, 1991; Del Casino et al., 1999). During generative cell division in Ornithogalum virens, metaphase spindle has a conventional shape, encompassing diffuse poles (Banaś et al., 1996). Focused spindle poles are typical for divisions during microsporogenesis and cultured plant cells. Such loose organization of the spindle poles is crucial for spindle plasticity, required to counteract spatial constrains due to the presence of the rigid cell wall. Moreover, the focused spindle pole helps to maintain normal chromosome distribution and grouping, because if the spindle pole is not convergent, the division may lead to several developmental defects, such as the formation of micronuclei, multiple micro-spindles and phragmoplasts. Therefore, in most cells, anaphase chromosome movement occurs concomitantly with transformation of broad and ill-defined polar areas into convergent ones. It seems that dynamic changes of the spindle pole during metaphase-anaphase transition occur only in cells with broad metaphase spindle (Fig. 2 e-g), while tapered spindles retain their shape throughout division. Notably, reversed spindle pole transformations take place during prophase-metaphase transition, when pointed poles transform into diffuse ones (Fig. 1 d-f, 2 c-e). Chromosomes/kinetochores may play an important role in this process, because the changes in spindle pole configuration are triggered after the nuclear envelope breakdown at the onset of prometaphase.

\subsection{The stages of spindle formation}

The pathways of spindle assembly depend on the presence/absence of the PPB. In cells with PPB, the microtubules first assemble perinuclear network, which gradually transforms into two dense accumulations or polar caps, located on both sides of the nucleus and oriented perpendicular to the PPB axis. The polar caps are linked by microtubules thus forming the initial, yet already bipolar, prophase spindle (Fig. $1 \mathrm{~d}$ ). Alternatively, the prophase spindle 
may exhibit an especially variable pattern of organization in cells lacking PPB (division of isolated endosperm cells, cultured suspension cells), but during prometaphase, the spindle ultimately acquires bipolar configuration. This pathway of spindle assembly is not well understood, but the initial stage is marked by the formation of dense microtubule network around the nucleus in early prophase (Fig. 2 b). In mid-late prophase, microtubule network transforms into bipolar (Fig. 2 c), multipolar or apolar spindle-like configurations (Smirnova and Bajer, 1998). After nuclear envelope breakdown, which marks the onset of prometaphase, aberrations of the spindle structure are eliminated/corrected and final alignment of spindle axis takes place (Fig. 2 d, e). Although multipolar prophase spindles are common, multipolar metaphase spindles are extremely rare in all types of dividing higher plant cells. Thus, the formation of the bipolar spindle in prophase is not a prerequisite for bipolarity of the metaphase spindle.

\subsection{MTOC-independent spindle assembly in higher plants}

It is no longer doubted that the functional spindle can be assembled by MTOC-independent mechanism (Murata et al., 2007), yet the suggestion that higher plant spindles are formed by self-reorganization of microtubules in the absence of the centrosome/MTOC, was made before substantial molecular evidence came out (Smirnova and Bajer, 1992). Observations on microtubule reorganization in isolated dividing endosperm cells and cell fragments of Scadoxus revealed that microtubule system is comprised of elementary structural and functional units termed microtubule converging centers (MTCC) (Bajer \& Molè-Bajer, 1986; Smirnova \& Bajer, 1994, 1998). The remarkable cycle of MTCCs formation, exceptionally manifested in endosperm cells, is an expression of self-reorganization of microtubules, and the self-reorganization is also the major "driving force" for spindle assembly. Conceptual importance of this conclusion was that (1) it argued against the role of centrosome/MTOC in spindle organization and (2) invited speculations that MTCCs might have been developed in higher plants as a functional substitute of localized MTOC(s). In view of this concept, the consecutive stages of spindle organization were defined. Microtubules/MTCCs growth is initiated from the nuclear surface, with minus ends attached or directed toward the nucleus. Excessive formation of MTCCs in prophase would require disconnection or severing of assembled MTCCs from the nucleating sites. Free MTCCs coalesce around the nucleus making dense perinuclear cage. Closely located MTCCs interact according to the dynamic properties of their ends and action of microtubule-based motors (self-reorganize). Random translocation and reorientation of MTCCs result in appearance of microtubules arrays of mixed polarity, followed by sorting into bundles of uniform polarity. The metaphase spindle is composed of microtubules with minus ends located away from the nucleus/chromosomes (oppositely to interphase), therefore during spindle assembly minus ends must be extended away, cross-linked and converged into poles, while plus ends are captured and transiently stabilized by chromosomes/kinetochores. Thus, primary, yet not necessarily bipolar, spindle is formed in prophase. In prometaphase prophase spindle transforms into ultimate bipolar metaphase spindle due to the interference of the chromosomes/kinetochores in this process. Simultaneously, the focused appearance of the prophase spindle poles is usually disturbed. The splitting of the spindle pole into multiple sub-poles reflects the potential functional autonomy of each K-fiber. This is crucial in conditions of constrained cellular geometry that precludes the movement/rotation of the spindle within the cell and change of the cell shape. Consequently, higher plants facilitate spindle formation by assembling MTCCs as ready-to-use units, instead of single 
microtubules. MTCCs form the spindle by self-reorganization therefore it is indeed "inside out" assembly of the spindle (Smirnova and Bajer, 1998).

The basic molecular mechanisms underlying MTOC-independent spindle assembly are now well understood (Wadsworth et al., 2010; Dunkan and Wakefiled, 2011). Briefly, in the initial phase of this process, chromosomes generate an environment enriched in RanGTP. Importins $\alpha$ and $\beta$ direct traffic through nuclear pores during interphase and sequester several essential proteins. Proteins binding to importins in mitosis are the kinesin-like protein XCTK2, targeting protein for Xklp2 (TPX2) and nuclear protein of the mitotic apparatus (NuMA). These proteins have been proposed to be targets of RanGTP in the spindle assembly pathway. The importins-TPX2 complex is dissociated by RanGTP and once released TPX2 promotes microtubule nucleation in the vicinity of chromosomes. The size and shape of chromatin defines the shape and orientation of the initial spindle (Dinarina et al., 2009). Assembled microtubules are first randomly oriented; then, they coalesce and start to organize through the movement and sorting of randomly nucleated microtubules into bipolar structures. Two proteins Eg5 and Kid were suggested to be regulated by RanGTP and to participate in these events. Sorted microtubules within each of the two arrays become bunched together at their minus ends, thus giving the entire array the shape of a spindle. Microtubule minus ends may be focus into spindle poles, and this process involves two proteins regulated by RanGTP: NuMA and XCTK2. The TPX2 was confirmed to be a central regulator of spindle assembly in plant cells, because its absence or excess inhibits spindle formation. Plant TPX2 is predominantly nuclear during interphase and is actively exported before nuclear envelope breakdown to initiate prophase spindle assembly. Thus, plants have adapted nuclear-cytoplasmic shuttling of TPX2 to maintain proper spindle assembly without centrosomes (Vos et al., 2008). However, there is a basic difference in microtubule focusing and pole stabilizing activities between vertebrate and plant cells. The convergent pole of the prophase spindle is rearranged into unfocused one during prophase-metaphase, and inversely during metaphase-anaphase (part 4.1), indicating that the mechanisms of microtubule focusing and stabilization are switched on and off throughout mitosis. In conclusion, MTOC-independent spindle assembly appears to be exceptionally manifested in higher plants, yet our knowledge about the molecular mechanisms of this process is still fragmentary.

\section{Phragmoplast}

Cytokinesis in cells of flowering plants is achieved through the construction of a new cell wall from the inside-out. A cell plate forms between daughter nuclei at the end of mitosis and expands centrifugally to form a new cell wall positioned between new plasma membranes. It is initiated through the fusion of Golgi-derived vesicles, which contribute polysaccharides, proteins and membranous vesicles for the cell plate formation. These vesicles are guided to the cell plate by the microtubules of the phragmoplast, assembled in the interzonal area after chromosome's segregation in anaphase. Therefore cytokinesis in plants is considered as targeted secretion to the plane of division (Assaad, 2001; Jürgens, 2005; Müller et al., 2009). Phragmoplast is composed of two sets of interacting microtubules of opposite polarities, terminating in ribosome free, cell plate assembly matrix, actin filaments, ER and Golgi derived vesicles. It begins to form in the central part of the intersone already in anaphase, expanding laterally throughout telophase. Upon completion of cytokinesis it fuses with the parental cell wall at the site of the PPB location, where the cell plate attaches to the mother cell wall. 


\subsection{The phragmoplast assembly and development}

The phragmoplast is assembled from microtubules of opposing polarity, interacting with their plus ends and pointing toward the equatorial region. These microtubules are aligned perpendicular to the future site of the cell plate localization. The phragmoplast is initiated in the midzone of the equatorial region in anaphase, between the trailing chromosome arms, and is shaped as cylindrical or barrel-like structure. Golgi-derived vesicles are carried from opposite sides, along microtubules toward the plus ends, where they fuse and first form vesicular-tubular membranous network, which is then transformed into the cell plate. During initial stages of formation, the cell plate is associated with the delivering vesicles microtubules of the phragmoplast. As the cell plate grows and expands centrifugally towards the parental cell wall, microtubules in the mid zone disassemble and in turn, assemble at the phragmoplast periphery, along the edge of the growing cell plate, resulting in displacement of microtubules toward the cell periphery. Consequently, the shape of the phragmoplast changes from cylindrical to ring-like, and at the end of cytokinesis two microtubule rings surround the edges of the newly formed cell plate. Microtubules of the phragmoplast provide the tracks for transport of vesicles to the site of the cell plate formation, yet the fusion of vesicles, development and maturation of the cell plate does not require microtubules. After the fusion of the cell plate with the parental cell wall, phragmoplast has an appearance of the microtubule ring, composed of the two sets of interacting antiparralel microtubules, and positioned at the site of the PPB localization. During specialized modes of cytokinesis, the phragmoplast is formed in cells dividing without a PPB, or even in syncytia between interphase nuclei, suggesting that the pathways of phragmoplast formations may vary among plant cells and tissues. For istance, during cellularization of endosperm, the multinucleate cytoplasm is reorganized into nuclear cytoplasmic domains defined by radial systems of microtubules, emanating from the nuclei. Adventitious phragmoplasts develop at the interfaces of microtubule systems emanating from adjacent nuclear cytoplasmic domains (Olsen et al., 1995), suggesting that microtubules of the spindle are not prerequisite for phragmoplast formation, and that phragmoplast-type of arrays may develop, where opposing sets of microtubules interact with their plus ends.

\subsection{The origin of the phragmoplast}

Phragmoplast is a ubiquitous plant structure, though the origin of phragmoplast microtubules and thereafter, the pathways of phragmoplast development and cell plate formation may differ among plant cells. For instance, in cells of apical meristem interzonal microtubules are abundant and give rise to the phragmoplast body (Staehelin \& Hepler, 1996), while the central region of interzone in dividing endosperm cells is often nearly devoid of microtubules (Bajer and Molè-Bajer, 1982). Instead, numerous microtubules, initiated at the polar areas, appear between trailing chromosome arms in mid-anaphase, gradually extend toward the equator and give rise to a phragmoplast (Bajer and Molè-Bajer, 1986). These observations demonstrate that phragmoplast originates either predominantly or entirely from new microtubules, growing from the polar areas, and suggest that plant cells may have evolved multiple mechanisms of phragmoplast assembly and development.

\subsubsection{Pathways of phragmoplast formation during "conventional" cytokinesis (i.e., in somatic plant tissues)}

"Conventional" cytokinesis follows after mitosis (Fig. 1 h-k), and phragmoplast originates from the interpolar microtubules of the anaphase spindle between the sister nuclei. 
Interpolar microtubules of each half-spindle interact in the midzone and establish antiparallel arrays, giving rise to the cylindrical microtubule bundle. This bundle differentiates into two sets of microtubules of opposing polarity - the phragmoplast. Microtubules deliver Golgi-derived vesicles towards the plus ends of microtubules, where vesicles fuse and form continuous vesicular-tubular network, localized between opposing microtubule sets. The vesicular-tubular network first differentiates into tubular network and then into fenestrated plate-like structure. The plate expands laterally, forming numerous finger-like projections that fuse with the parental cell membrane. This is followed by the closing of the plate fenestrae and beginning of the cellulose synthesis. The formation of membranous network takes place in association with the phragmoplast microtubules, while cell wall construction within this network occurs after the disassembly of microtubules (Samuels et al., 1995).

\subsubsection{Syncytial pathway of phragmoplast formation during "nonconventional" cytokinesis (i.e., during cellularization of nuclear endosperm)}

"Nonconventional" cytokinesis takes place when mitosis is not immediately followed by cytokinesis, and the new cell walls are formed at a later stage between sister and non sister nuclei. The cell plate formation is preceded by synchronous appearance of microtubule sets of opposing polarity, followed by the assembly of numerous mini-phragmoplasts between non sister nuclei. Each mini-phragmoplast consists of two opposing sets of microtubules, which originate from the overlapping microtubule clusters. Golgi-derived vesicles are transported along these microtubules, fuse with each other and generate a network of wide membranous tubes. Subsequently, these wide tubes undergo a series of transformations that eventually give rise to the mature cell wall (Otegui and Staehelin, 2000a, 2000b). Thus interpolar microtubules of the spindle are not prerequisite for phragmoplast assembly. The spindle-independent phragmoplast formation also takes place during microsporogenesis, when microtubules of the radial systems, emanating from the nuclei, elongate and interact with plus ends, forming phragmoplast-type of arrays (Olsen et al., 1995).

Moreover, interpolar microtubules do not seem to be involved in the assembly of the phragmoplast even in certain types of dividing cells, because very few or no interpolar microtubules may be present in the interzone of endosperm cells during anaphase (Fig. $2 \mathrm{~g}$ ). Therefore, these sets of spindle microtubules cannot contribute to the formation of the phragmoplast precursor (cylindrical microtubule bundle). Instead, numerous microtubules grow from the polar areas towards the cell periphery and the cell equator in anaphasetelophase, assembling aster-like microtubule configurations (Bajer and Mole-Bajer, 1986). The phragmoplast is assembled from the microtubules elongating from opposing polar areas and interacting in the mid zone (Fig. $2 \mathrm{~h}$, i). The initial phragmoplast is a barrel-like or double dome structure, and at final stages of cytokinesis it is shaped as a circular, closed and narrow band.

\subsection{Molecular components of the phragmoplast}

Proteins associated with the phragmoplast are usually grouped according to the functions during cytokinesis. The phragmoplast plays an essential role in the targeted delivery of membrane vesicles to the plane of cell division, predicted by the PPB. Membrane associated functions are carried by classes of proteins involved in vesicles docking, fusion, budding and construction of the membrane. Cytoskeleton associated functions of the phragmoplast are predetermined by the PPB (part 4.3 and 4.4), and both structures share certain classes of 
proteins, for instance, cross-linking and stabilizing of microtubules by MOR1/GEM 1 and MAP65 family members. A special class of cytokinetic kineisins (part 2.5.1) is directly involved in the organization and proper functions of the phragmoplast. Structural MAPs and kinesins regulate the dynamic stability of the microtubules in the phragmoplast body, maintain phragmoplast integrity and in this way provide tracks for targeted transport of vesicles and formation of the cell plate (Jürgens, 2005; Müller et al., 2009).

\section{Concluding remarks}

Extensive studies of plant microtubules for past decades led to the significant progress in understanding of the overall dynamics of plant microtubules, mechanisms underlying the nucleation, organization and function of cortical microtubule arrays, the specificity of plant cytokinesis and on the contrary, the unifying features with animal cytokinesis. The isolation, identification and characterization of vast family of microtubule associated proteins closed many gaps in our knowledge on the functional properties of plants microtubules and the regulation of their behavior. In spite of that, many questions remain unanswered, or may be simply overlooked. The goal of this chapter was to give an overview of basic and mainly structural aspects of plant microtubule cytoskeleton, which is, by all means, not complete due to space limitations, and draw attention to unsolved problems. Among the intriguing questions that remain are: the relationship between cortical microtubules and cellulose microfibrils orientation, the role of the radial microtubules and PPB in morphogenesis of the mitotic spindle, the molecular mechanisms of MTOC-independent plant spindle assembly as compared to other organisms, and even more, if one is enough curios and inquisitive to look inside the plant cell.

\section{Aknowledgements}

I would like to thank my colleague Wang Wenzhu (MSU) for help with the illustrations.

\section{References}

Ambrose, C. \& Cyr, R. (2008). Mitotic spindle organization by the preprophase band. Mol. Plant, 1, 950-960.

Assaad, F. (2001). Plant cytokinesis: exploring the link. Plant Physiol., 126, 509-516.

Bajer, A. \& Molè-Bajer, J. (1982). Asters, poles and transport properties within spindle-like microtubule arrays. Cold Spring Harbor Symp., 46, 263-283.

Bajer, A. \& Molè-Bajer, J. (1986). Reorganization of microtubules in endosperm cells and cell fragments of the higher plant Haemanthus in vivo. J. Cell Biol., 102, 263-281.

Banaś, M., Tirlapur, U., Charzyńska, M. \& Cresti, M. (1996). Some events of mitosis and cytokinesis in the generative cell of Ornithogalum virens L. Planta, 199, 202-208.

Bisgrove, S., Hable, W. \& Kropf, D. (2004). +TIPs and microtubule regulation. The beginning of the plus ends in plants. Plant Physiol., 136, 3855-3863.

Buschmann, H., Chan, J., Sanchez-Pulido, L., Andrade-Navarro, M., Doonan, J. \&, Lloyd, C. (2006). Microtubule-associated AIR9 recognizes the cortical division site at preprophase and cell-plate insertion. Curr. Biol., 16, 1938-1943.

Cai, G. (2010). Assembly and disassembly of plant microtubules: tubulin modifications and binding to MAPs. Exp. Botany, 61, 623-626. 
Crowell, E., Bischoff, V., Desprez, T., Rolland, A., Stierhof, Y., Schumacher, K., Gonneau, M., Höfte, H. \& Vernhettes, S. (2009). Pausing of Golgi bodies on microtubules regulates secretion of cellulose synthase complexes in Arabidopsis. Plant Cell, 21, 1141-1154.

Del Casino, C., Bohdanowitcz, J., Lewandowska, B. \& Cresti, M. (1999). The organization of microtubules during generative-cell division in Convallaria majalis. Protoplasma, 207, 147-153.

Dinarina, A., Pugieux, C., Corral, M., Loose, M., Spatz, J., Karsenti, E. \& Nédélec, F. (2009). Chromatin shapes the mitotic spindle. Cell, 138, 502-512.

Dhonukshe, P., Mathur, J., Hulskamp, M. \& Gadella, T. (2005). Microtubule plus-ends reveal essential links between intracellular polarization and localized modulation of endocytosis during division-plane establishment in plant cells. BMC Biol., 3, 11.

Duncan, T. \& Wakefield, J. (2011). 50 ways to build a spindle: the complexity of microtubule generation during mitosis. Chromosome Res., 19, 321-333.

Eigsti, O. \& Dustin, P. (1955). Colchicine in agriculture, medicine, biology and chemistry. Iowa State Coll. Press, Aimes, Iowa.

Ehrhardt, D. (2008). Straighten up and fly right - microtubule dynamics and organization of non-centrosomal arrays in higher plants. Curr. Opin. Cell Biol., 20, 107-116.

Erhardt, M., Stoppin-Mellet, V., Campagne, S., Canaday, J., Mutterer, J., Fabian, T., Sauter, M., Muller, T., Peter, C., Lambert, A-M. \& Schmit, A-C. (2002). The plant Spc98p homologue colocalizes with $\gamma$-tubulin at microtubule nucleation sites and is required for microtubule nucleation. J. Cell Sci., 115, 2423-2431.

Evrard, J-L., Pieuchot, L., Vos, J., Vernos, I. \& Schmit, A-C. (2009). Plant TPX2 and related proteins. Plant Sign. Behav., 4, 69-72.

Fache, V., Gaillard, J., Van Damme, D., Geelen, D., Neumann, E., Stoppin-Mellet, V. \& Vantard, M. (2010) Arabidopsis kinetochore fiber-associated MAP65-4 cross-links microtubules and promotes microtubule bundle elongation. Plant Cell, 22, 38043815.

Fosket, D. \& Morejohn, L. (1992). Structural and functional organization of tubulin. Annu. Rev. Plant Physiol. Plant Mol. Biol., 43, 201-240.

Hashimoto, T. \& Kato, T. (2006). Cortical control of plant microtubules. Curr. Opin. Plant Biol., 9, 5-11.

Heald, R., Tournebize, R., Blank, T., Sandaltzopoulos, R., Becker, P., Hyman, A. \& Karsenti, E. (1996). Self-organization of microtubules into bipolar spindle around artificial chromosomes in Xenopus egg extracts. Nature, 382, 420-425.

Heald, R., Tournebize, R., Habermann, A., Karsenti, E. \& Hyman, A. (1997). Spindle assembly in Xenopus egg extracts: respective roles of centrosomes and microtubule self-organization. J. Cell Biol., 138, 615-628.

Hyams, J. (1985). Binding of Tetrahymena dynein to axonemes of Marsilea vestita lacking the outer dynein arms. J Cell Sci., 73, 299-310.

Jürgens, G. (2005). Plant cytokinesis: fission by fusion. Trends Cell Biol., 15, 277-283.

Kaloriti, D., Galva, C., Parupalli, C., Khalifa, N., Galvin, M. \& Sedbrook, J. (2007). Microtubule associated proteins in plants and the processes they manage. J. Inegrative Plant Biol., 49, 1164-1173. 
Komaki, S., Abe, T., Coutuer, S., Inzé, D., Russinova, E. \& Hashimoto, T. (2010). Nuclearlocalized subtype of end-binding 1 protein regulates spindle organization in Arabidopsis. J. Cell Sci., 123, 451-459.

Lee, Y. \& Liu, B. (2004). Cytoskeletal motors in Arabidopsis. Sixty one kinesins and seventeen myosins. Plant Physiol., 136, 3877-3883.

Liu, B. \& Palevitz, B. (1991). Kinetochore fiber formation in dividing generative cells of Tradescantia. Kinetochore reorientation associated with the transition between lateral microtubule interactions and end-on kinetochore fiber. J. Cell Sci., 98, 475482.

Liu, B., Joshi, H., Wilson, T., Silflow, C., Palevitz, B. \& Snustad, D. (1994). $\gamma$-tubulin in Arabidopsis: gene sequence, immunoblot, and immunofluorescence studies. Plant Cell, 6, 303-314.

Lucas, J. \& Shaw, S. (2008) Cortical microtubule arrays in the Arabidopsis seedlings. Curr. Opin. Plant Biol., 1, 94-98.

Mineyuki, Y. (1999). The preprophase band of microtubules: its function as a cytokinetic apparatus in higher plants. Int. Rev. Cytol., 187, 1-49.

Mineyuki, Y. (2007). Plant microtubule studies: past and present. J. Plant Res., 120, 4551.

Morejohn, L. \& Fosket, D. (1991). The biochemistry of compounds with anti-microtubule activity in plant cells. Pharmac. Ther., 51, 217-230.

Moscatelli, A., Del Casino, C., Lozzi, L., Cai, G., Scali, M., Tiezzi, A. \& Cresti, M. (1995). High molecular weight polypeptides related to dynein heavy chains in Nicotiana tabacum pollen tubes J. Cell Sci., 108, 1117-1125.

Müller, S., Wright, A. \& Smith, L. (2009). Division plane control in plants: new players in the band. Trends Cell Biol., 19, 180-188.

Murata, T., Sonobe, S., Baskin, T., Hyodo, S., Hasezawa, S., Nagata, T., Horio, T. \& Hasebe, M. (2005) Microtubule-dependent microtubule nucleation based on recruitment of Y-tubulin in higher plants. Nature Cell Biol., 7, 961-968.

Murata, T. \& Hasebe, M. (2007). Microtubule-dependent microtubule nucleation in plant cells. J. Plant Res., 120, 73-78.

Murata, T., Tanahashi, T., Nishiyama, T., Yamaguchi, K. \& Hasebe, M. (2007). How do plants organize microtubules without a centrosome. J. Inegrtive. Plant Biol., 49, 11541163.

Olsen, O-A., Brown, R. \& Lemmon, B. (1995). Pattern and process of wall formation in developing endosperm. BioEssays, 17, 803-812.

Otegui, M. \& Staehelin, A. (2000a). Syncytial-type cell plates: a novel kind of cell plate involved in endosperm cellularization of Arabidopsis. Plant Cell, 12, 933-947.

Otegui, M. \& Staehelin, A. (2000b). Cytokinesis in flowering plants: more than one way to divide the cell. Curr. Opin. Plant Biol., 3, 493-502.

Paredez, A., Wright, A. \& Ehrhardt, D. (2006). Microtubule cortical array organization and plant cell morphogenesis. Curr. Opin. Plant Biol., 9, 571-578.

Paredez, A., Persson, S., Ehrhardt D., Sommerville, C. (2008). Genetic evidence that cellulose synthase activity influence microtubule cortical array organization. Plant Physiol., $147,1723-1734$.

Pastuglia, M. \& Bouchez, D. (2007). Molecular encounters at microtubule ends in the plant cortex. Curr. Opin. Plant Biol., 10, 1-7. 
Rasmussen, C., Humphries, J. \& Smith L. (2011). Determination of symmetric and asymmetric division planes in plant cells. Annu. Rev. Plant Cell Biol. 62, 387-409.

Renzaglia, K. \& Garbary, D. (2001). Motile gametes of land plants: diversity, development and evolution. Crit. Rev. Plant Sci., 20, 107-213.

Samuels, A., Giddings, T. \& Staehelin, L. (1995). Cytokinesis in tobacco BY-2 and root tip cells: a new model of cell plate formation in higher plants. J. Cell Biol., 130, 13451357.

Sedbrook, J. (2004). MAPs in plant cells: delineating microtubule growth dynamics and organization. Curr. Opin. Plant Biol., 7, 632-640.

Shanina, N., Lazareva, E., Skorova, E., Chentsov, Y. \& Smirnova, E. (2009). A high molecular weight polypeptide cross-reacting with the antibodies to the dynein heavy chain localizes to the subset of Golgi complex in higher plant cells. Cell Biol. Intern., 33, 290-300.

Shaw, S., Kamyar, R. \& Ehrhardt, D. (2003). Sustained microtubule treadmilling in Arabidopsis cortical arrays. Science, 300, 1715-1718.

Smirnova, E. \& Bajer, A. (1992). Spindle pole in higher plant mitosis. Cell Motil. Cytoskel., 23, $1-7$.

Smirnova, E. \& Bajer, A. (1994). Microtubule converging centers, reorganization of the interphase cytoskeleton and the mitotic spindle in higher plant Haemanthus. Cell Motil. Cytoskel., 27, 219-233.

Smirnova, E. \& Bajer, A. (1998). Early stages of spindle formation and independence of chromosome and microtubule cycles in Haemanthus endosperm. Cell Motil. Cytoskel., 40, 22-37.

Staehelin, A. \& Hepler, P. (1996). Cytokinesis in higher plants. Cell, 84, 821-824.

Van Damme, D., Vanstraelen, M. \& Geelen, D. (2007). Cortical division zone establishment in plant cells. Trends Plant Sci., 12, 458-464.

Van Damme, D. \& Geelen, D. (2008). Demarcation of the cortical division zone in dividing plant cells. Cell Biol. Int., 32, 178-187.

Vaughn, K. \& Renzaglia K. (2006) Structural and immunocytochemical characterization of the Ginkgo biloba L. sperm motility apparatus. Protoplasma, 227, 165-173.

Vos, J., Pieuchot, L., Evrard, J-L., Janski, N., Bergdoll, M., de Ronde, D., Perez, L., Sardon, T., Vernos, I. \& Schmit A-C. (2008). The plant TPX2 protein regulates prospindle assembly before nuclear envelope breakdown. Plant Cell, 20, 2783-2797.

Wadsworth, P., Lee, W-L., Murata, T. \& Baskin, T. (2011). Variations on theme: spindle assembly in diverse cells. Protoplasma, 248, 439-446.

Wasteneys, G. (2004). Progress in understanding the role of microtubules in plant cells. Curr. Opin. Plant Biol., 7, 651-660.

Wasteneys, G. \& Ambrose J. (2008). Spatial organization of plant cortical microtubules: close encounters of the 2D kind. Trends Cell Biol., 19, 62-71.

Xu, X., Zhao, Q., Rodrigo-Peiris, T., Brkljacic, J., He, C., Müller, S. \& Meier, I. (2008). RanGAP1 is a continuous marker of the Arabidopsis cell division plane. Proc. Natl. Acad. Sci. USA, 105, 18637-18642. 


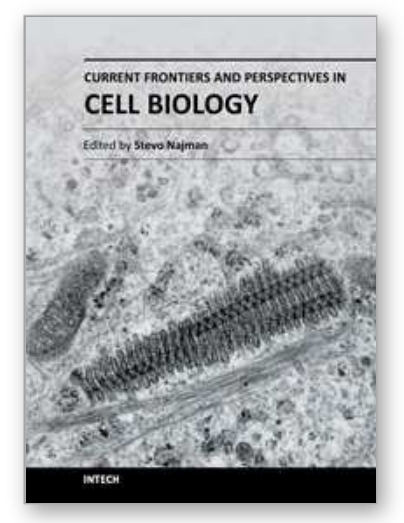

\section{Current Frontiers and Perspectives in Cell Biology \\ Edited by Prof. Stevo Najman}

ISBN 978-953-51-0544-2

Hard cover, 556 pages

Publisher InTech

Published online 25, April, 2012

Published in print edition April, 2012

\section{How to reference}

In order to correctly reference this scholarly work, feel free to copy and paste the following:

Elena Smirnova (2012). Microtubules During the Cell Cycle of Higher Plant Cells, Current Frontiers and Perspectives in Cell Biology, Prof. Stevo Najman (Ed.), ISBN: 978-953-51-0544-2, InTech, Available from: http://www.intechopen.com/books/current-frontiers-and-perspectives-in-cell-biology/microtubules-during-thecell-cycle-of-higher-plant-cells

\section{INTECH}

open science | open minds

\section{InTech Europe}

University Campus STeP Ri

Slavka Krautzeka 83/A

51000 Rijeka, Croatia

Phone: +385 (51) 770447

Fax: +385 (51) 686166

www.intechopen.com

\section{InTech China}

Unit 405, Office Block, Hotel Equatorial Shanghai

No.65, Yan An Road (West), Shanghai, 200040, China

中国上海市延安西路65号上海国际贵都大饭店办公楼405单元

Phone: +86-21-62489820

Fax: +86-21-62489821 
(C) 2012 The Author(s). Licensee IntechOpen. This is an open access article distributed under the terms of the Creative Commons Attribution 3.0 License, which permits unrestricted use, distribution, and reproduction in any medium, provided the original work is properly cited. 